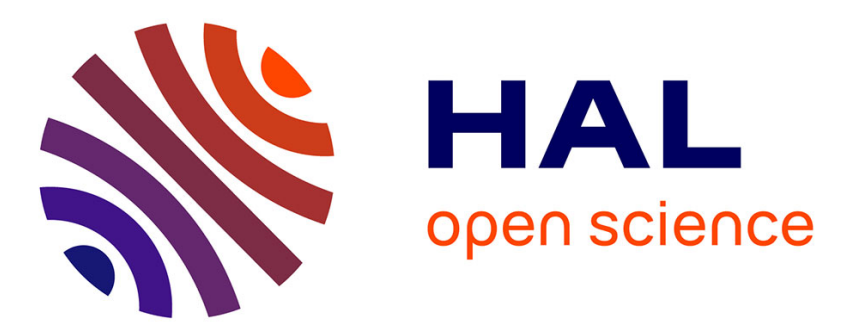

\title{
Détection des jets moléculaires modulés : étude de la réponse en fonction des caractéristiques géométriques du détecteur
}

C. Genty, J.G. Théobald

\section{- To cite this version:}

C. Genty, J.G. Théobald. Détection des jets moléculaires modulés : étude de la réponse en fonction des caractéristiques géométriques du détecteur. Revue de Physique Appliquée, 1973, 8 (4), pp.361-366. 10.1051/rphysap:0197300804036100 . jpa-00243695

\section{HAL Id: jpa-00243695 https://hal.science/jpa-00243695}

Submitted on 1 Jan 1973

HAL is a multi-disciplinary open access archive for the deposit and dissemination of scientific research documents, whether they are published or not. The documents may come from teaching and research institutions in France or abroad, or from public or private research centers.
L'archive ouverte pluridisciplinaire HAL, est destinée au dépôt et à la diffusion de documents scientifiques de niveau recherche, publiés ou non, émanant des établissements d'enseignement et de recherche français ou étrangers, des laboratoires publics ou privés. 


\title{
DÉTECTION DES JETS MOLÉCULAIRES MODULÉS : ÉTUDE DE LA RÉPONSE EN FONCTION DES CARACTÉRISTIQUES GÉOMÉTRIQUES DU DÉTECTEUR
}

\author{
C. GENTY et J. G. THÉOBALD \\ Laboratoire de Spectroscopie Hertzienne et d'Electronique \\ Faculté des Sciences, 25030 Besançon, France
}

(Reçu le 7 mai 1973)

\begin{abstract}
Résumé. - On étudie le signal fourni par un détecteur recevant un jet modulé en intensité. Le détecteur se comporte comme un intégrateur (circuit $R C$ ) et on trace la courbe de réponse en fonction de la fréquence de modulation. Les courbes de rapport signal sur bruit permettent de déterminer les meilleures conditions de travail.

Abstract. - We study the signal from a detector exposed to a molecular beam of modulated intensity.

The detector behaves like an integrator ( $R C$ circuit) and we study the signal as a function of the modulation frequency. The curve giving the signal to noise ratio leads us to determine the best working conditions.
\end{abstract}

1. Introduction. - Les techniques de jet moléculaire connaissent actuellement un développement accru et nombreuses sont les méthodes qui passent par la détection du jet, tant dans le domaine de l'étude thermodynamique des jets [1], [2], [3], [4], que dans les applications les plus diverses, en spectroscopie par exemple [5]. On est naturellement conduit à rechercher des dispositifs de détection de sensibilité toujours croissante; or il va de soi que toutes les méthodes actuellement employées conduisent à une destruction du jet moléculaire et, partant, à une certaine accumulation des molécules au niveau du système détecteur. L'état d'équilibre n'est donc atteint qu'avec une certaine constante de temps et si ce fait est sans importance lorsqu'on détecte un jet continu, il en va tout autrement lorsqu'on travaille sur un jet d'amplitude variable, la forme des variations étant modifiée par l'effet d'intégration dû à la constante de temps.

Nous nous proposons d'étudier ce problème pour un détecteur constitué d'une jauge à ionisation placée dans une cavité de volume $V$ (cavité de détection) communiquant avec le jet par un tube cylindrique de longueur $l$ et de rayon $r$, placé sur l'axe du jet (Fig. 1). Nous travaillons sur un jet de méthanal modulé en amplitude, soit sinusoïdalement, soit en créneau.

2. Appareillage et méthode expérimentale. - Nous utilisons un spectromètre à jet moléculaire [6] conçu pour détecter directement les transitions par l'intermédiaire des variations de l'intensité du jet. Pour ce qui nous concerne, l'intérêt de ce dispositif, dont la figure 2 donne le schéma de principe, est de permettre très facilement une modulation partielle du jet de forme et de fréquence quelconque. La molécule de méthanal possède un moment dipolaire électrique et des doublets du type K. De ce fait, le 1 er focaliseur (du type électrostatique multipolaire) élimine les molécules (issues d'une source fonctionnant par effusion) appartenant aux niveaux inférieurs des doublets, tandis que les molécules appartenant aux niveaux d'énergie supérieurs sont focalisées.

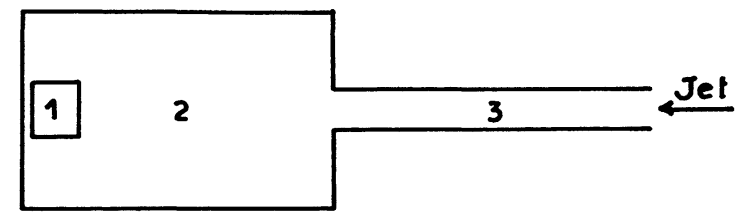

FIG. 1. - Détecteur :

1) Jauge à ionisation.

2) Cavité de détection.

3) Tube d'entrée.

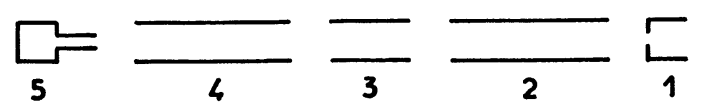

FIG. 2. - Schéma de principe du spectromètre :

1) Source.

2) $1^{\text {er }}$ focaliseur électrostatique.

3) Cellule d'irradiation.

4) $2^{\mathrm{e}}$ focaliseur électrostatique.

5) Détecteur. 
Si au niveau de la cellule d'irradiation (condensateur plan) est appliqué un champ électrique HF dont la fréquence est égale à la fréquence de transition d'un doublet $\mathrm{K}$, les molécules appartenant à ce doublet vont transiter dans l'état inférieur. Elles seront ensuite éliminées par le $2^{\mathrm{e}}$ focaliseur (identique au $1^{\mathrm{er}}$ ) et il se produira au niveau du détecteur une diminution de l'intensité du jet.

Il est clair que si le champ HF est modulé, on aura à l'entrée du détecteur un jet modulé, la forme et la fréquence de cette modulation reproduisant celle du champ électrique (Fig. 3 pour une modulation créneau).

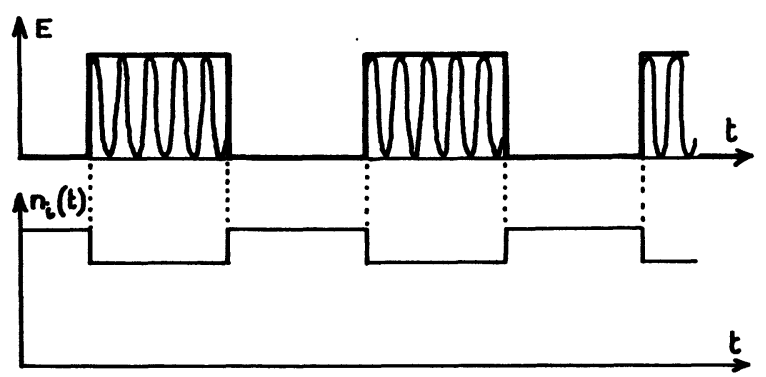

FIG. 3. - Modulation du jet par le champ HF : Correspondance entre le champ électrique $E$ appliqué sur la cellule d'irradiation et l'intensité du jet $\left[n_{1}(t)\right]$ à l'entrée du détecteur.

Il convient de faire deux remarques au sujet de cette technique de modulation :

$1^{\circ} \mathrm{La}$ distance cellule d'irradiation-détecteur doit être suffisamment courte pour que la déformation consécutive à la répartition des vitesses soit négligeable.

Cette circonstance est réalisée ici avec une distance de l'ordre de $30 \mathrm{~cm}$.

$2^{\circ}$ La cellule d'irradiation se comporte comme un amplificateur, le jet ne reproduisant la forme de la HF que dans la mesure où la courbe de réponse (c'est-àdire intensité de la raie de résonance en fonction du niveau HF appliqué sur la cellule) est linéaire.

La figure 4 représente cette courbe de réponse dans nos conditions expérimentales : cellule de longueur $15 \mathrm{~cm}$, écartement des plaques de condensateur $1 \mathrm{~cm}$; transition $4_{3}$ de fréquence voisine de $4,573 \mathrm{MHz}$.

La limitation d'amplitude de la HF est impérative dans le cas d'une modulation sinusoïdale, le champ HF devant être tel que l'on se situe dans la partie

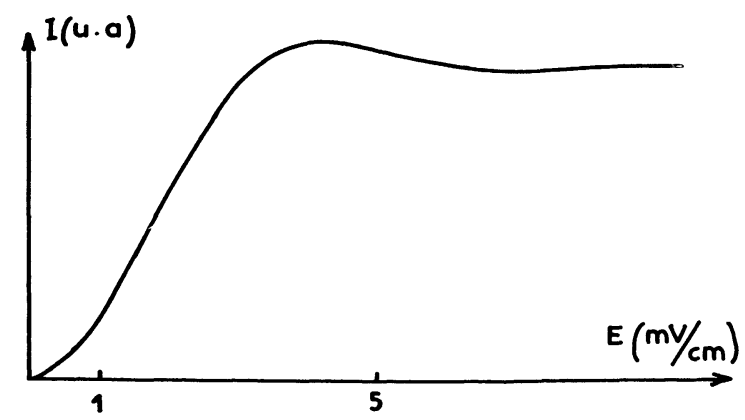

Fig. 4. - Intensité de la raie 43 en fonction de l'amplitude du champ HF. linéaire de la courbe. Par contre, elle est pratiquement sans objet dans le cas d'une modulation créneau car la probabilité de transition attachée au temps de montée du créneau est négligeable.

La jauge à ionisation (Edwards IG 2 MA) placée dans la cavité de détection fournit un signal électrique proportionnel à la pression. On effectue une détection synchrone de ce signal (PAR, modèle 124) avec la référence pilotant la modulation du champ HF. On détecte en conséquence le fondamental du signal fourni par le détecteur de jet.

3. Réponse du détecteur à un jet d'intensité variable. - 3.1 Equation DE FonCtionNement. - Le signal fourni par le détecteur est proportionnel à la pression dans la cavité de détection, c'est-à-dire proportionnel au nombre de molécules par unité de volume dans cette cavité. Soit $N(t)$ ce nombre au temps $t$.

Pour un jet d'intensité variable, le nombre de molécules arrivant par seconde sur le détecteur est une fonction du temps, soit $n_{\mathrm{i}}(t)$.

D'après les résultats classiques [7] le nombre de molécules émergeant du détecteur par seconde est proportionnel à $N(t)$ soit :

$$
n_{\mathrm{s}}(t)=A N(t) \quad \text { avec } \quad A=\frac{1}{\kappa} \frac{1}{4} \mathrm{vS} ;
$$

$\kappa=$ facteur de Clausing du tube d'entrée,

$v=$ vitesse moyenne des molécules,

$\mathrm{S}=$ section du tube d'entrée.

Remarques. - On suppose tout le système en équilibre de température.

- La pression ambiante moyenne $\left(P \simeq 10^{-6}\right.$ torr dans nos expériences) est telle que le libre parcours moyen est très supérieur à 1 , si bien que toutes les molécules du jet pénètrent dans la cavité de détection quel que soit $N(t)$.

$N(t)$, et par conséquent le signal fourni par le détecteur, obéit donc à l'équation :

$$
V \frac{\mathrm{d} N(t)}{\mathrm{d} t}+A N(t)=n_{\mathrm{i}}(t) .
$$

On remarque que cette équation est caractéristique d'un intégrateur, un circuit $R C$ par exemple, le volume $V$ de la cavité de détection jouant le rôle de la capacité $C$, la constante $A$, liée au tube d'entrée, étant analogue à la conductance $1 / R$, le système ayant une constante de temps $\tau=V / A$.

Cette équation n'est valable que si la répartition des molécules dans la cavité est parfaitement homogène. Si on veut pouvoir considérer le détecteur comme un intégrateur de signaux, cette condition impose une limitation aux géométries possibles de ce dernier comme on le verra plus loin.

3.2 CAS D'UNe modulation SINUSOÏDAle - Le champ HF étant modulé sinusoïdalement à la fré- 
quence $v=\omega / 2 \pi, n_{\mathrm{i}}(t)$ a la forme donnée par la figure 5. La modulation n'étant que partielle, il existe un fond continu correspondant à $N_{0}$. L'existence de ce fond continu est sans incidence sur les résultats et

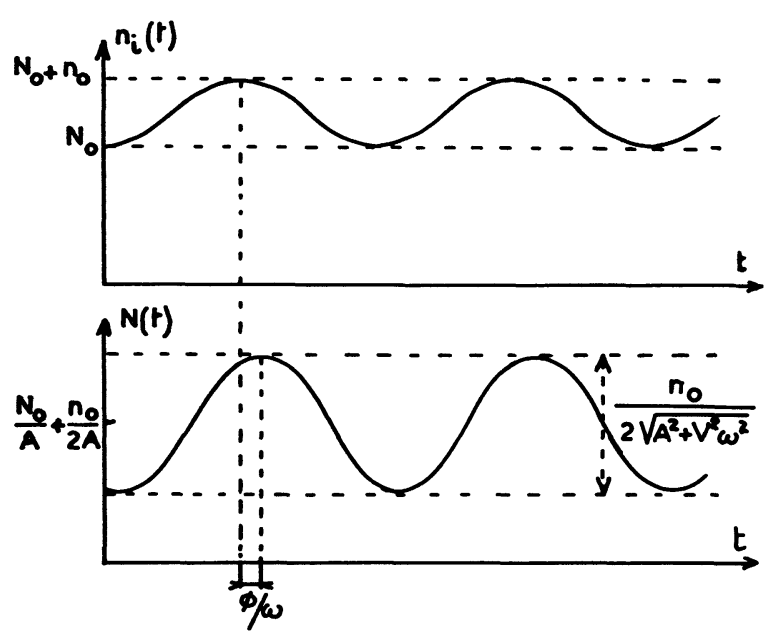

FIG. 5. - Modulation sinusoïdale.

seule intervient la partie dépendant du temps. Nous écrivons donc :

$$
n_{\mathrm{i}}(t)=\frac{n_{0}}{2}(1-\cos \omega t)
$$

L'évolution du nombre de molécules dans la cavité de détection est alors donnée par :

$N(t)=C \mathrm{e}^{-A t / V}+\frac{n_{0}}{2 A}-\frac{n_{0}}{2 \sqrt{A^{2}+V^{2} \omega^{2}}} \cos (\omega t-\varphi)$

avec

$$
\operatorname{tg} \varphi=\frac{V \omega}{A}
$$

Une fois le régime permanent établi, le détecteur de jet fournit donc un signal utile (ramené à sa partie périodique) sinusoïdal d'amplitude $\frac{n_{0}}{2 \sqrt{A^{2}+V^{2} \omega^{2}}}$ et déphasé de $\varphi$ par rapport au signal d'entrée $n_{\mathrm{i}}(t)$ (Fig. 5).

L'intensité du signal recueilli après détection synchrone est donc :

$$
I_{\mathrm{s}}=\alpha \frac{n_{0}}{2 \sqrt{A^{2}+V^{2} \omega^{2}}},
$$

$\alpha$ étant un facteur de proportionnalité.

3.3 CAS D'une modulation CRÉneau. - Soit maintenant un champ HF modulé en créneau à la fréquence $v$ (période $T$ ). $n_{\mathrm{i}}(t)$ s'écrit alors

$$
\begin{array}{rlrlrl}
n_{\mathrm{i}}(t) & =n_{0} & \text { pour } & 0 \leqslant t \leqslant T / 2 \\
& =0 & \text { pour } & & T / 2 \leqslant t \leqslant T .
\end{array}
$$

$N(t)$ devient :

$N(t)=\frac{n_{0}}{A}\left[1-\frac{e^{x}-1}{e^{x}-e^{-x}} \mathrm{e}^{-A t / V}\right] \quad$ pour $\quad 0 \leqslant t \leqslant \frac{T}{2}$

$$
=\frac{n_{0}}{A}\left[\frac{e^{x}-1}{e^{x}-e^{-x}} e^{x} \mathrm{e}^{-A t / V}\right] \text { pour } \quad \frac{T}{2} \leqslant t \leqslant T
$$

avec

$$
x=\frac{A}{V} \frac{\pi}{\omega}
$$

Le signal fourni par le détecteur de jet (proportionnel à $N(t))$ a la forme de la figure 6 .

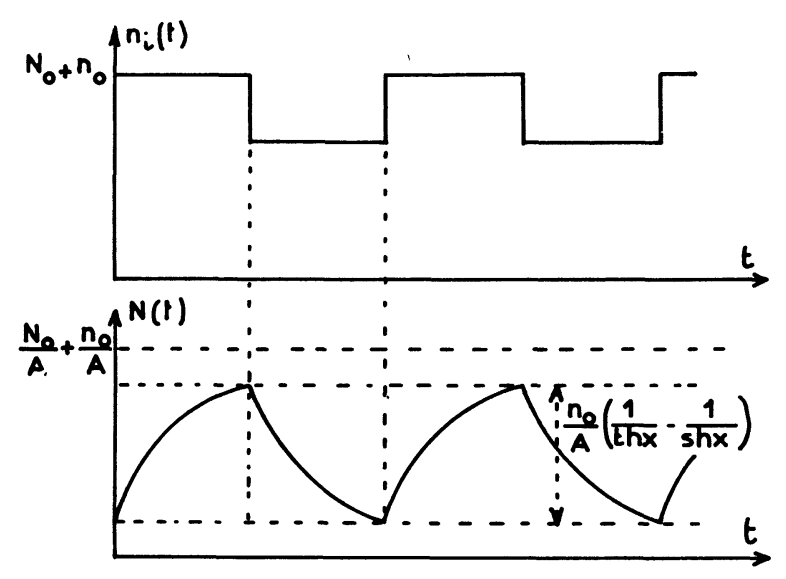

Fig. 6. - Modulation créneau.

Sous forme de la somme de ses composantes de Fourier, $N(t)$ s'écrit :

$$
\begin{array}{r}
N(t)=\frac{n_{0}}{2 A}-\frac{2}{\pi} \sum_{k} \frac{n_{0}}{(2 k+1) \sqrt{A^{2}+(2 k+1)^{2} V^{2} \omega^{2}}} \times \\
\times \sin \left[(2 k+1) \omega t-\varphi_{2 k+1}\right]
\end{array}
$$

avec

$$
\operatorname{tg} \varphi_{2 k+1}=(2 k+1) \frac{V \omega}{A}
$$

L'intensité du signal recueilli après détection synchrone, soit $I_{\mathrm{c}}$, est proportionnelle à l'amplitude du fondamental, soit :

$$
I_{\mathrm{c}}=\alpha-\frac{4}{\pi} \frac{n_{0}}{2 \sqrt{A^{2}+V^{2} \omega^{2}}}=\frac{4}{\pi} I_{\mathrm{s}}
$$

ce qui traduit le résultat tout à fait classique du déphasage par intégration d'un signal sinusoïdal.

4. Résultats expérimentaux. - Nous comparons les résultats donnés par 4 détecteurs de géométrie différentes.

Pour les tubes d'entrée employés $(r \ll l)$ le facteur de Clausing est donné [7] par :

$$
\frac{1}{\kappa}=\frac{8}{3} \frac{r}{l}
$$


si bien que :

$$
A=\frac{2}{3} \pi \mathbf{v} \frac{r^{3}}{l}
$$

Tous les tubes ont le même rayon : $r=0,5 \mathrm{~cm}$.

$v=600 \mathrm{~m} / \mathrm{s}$ déterminé sur le spectromètre par une expérience antérieure [8].

Afin de pouvoir comparer les différents détecteurs, toutes les conditions expérimentales sont maintenues rigoureusement constantes.

Pression ambiante $\simeq 10^{-6}$ torr.

Tension de focalisation : $10 \mathrm{kV}$.

Pression source : 1 torr.

Amplitude du champ HF : 7,5 mV/cm (modulation créneau), $2,5 \mathrm{mV} / \mathrm{cm}$ (modulation sinusoïdale).

Les 2 dernières données définissent $n_{0}$.

Les détecteurs ont les caractéristiques suivantes :

- détecteur $1: V=92 \mathrm{~cm}^{3}, l=20 \mathrm{~cm}, \tau=117 \mathrm{~ms}$,

- détecteur 2: $V=92 \mathrm{~cm}^{3}, l=10 \mathrm{~cm}, \tau=58,5 \mathrm{~ms}$,

- détecteur $3: V=53 \mathrm{~cm}^{3}, l=20 \mathrm{~cm}, \tau=67,5 \mathrm{~ms}$,

- détecteur $4: V=53 \mathrm{~cm}^{3}, l=10 \mathrm{~cm}, \tau=33,7 \mathrm{~ms}$.

4.1 RÉPONSE EN FONCTION DE LA FRÉQUENCE DE MODULATION. - Le champ HF étant modulé en

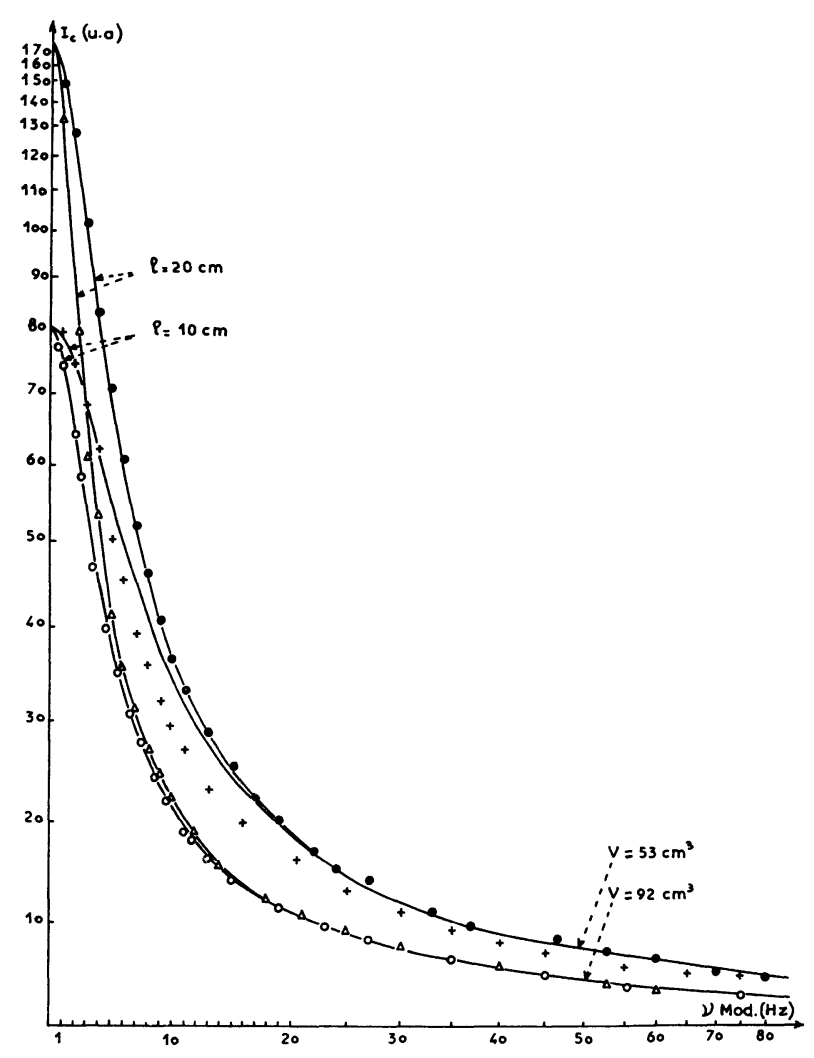

FIG. 7. - Réponse en fonction de la fréquence pour une modulation en créneau.

Points expérimentaux $\triangle$ : détecteur 1.

$$
\begin{aligned}
& O \text { : détecteur } 2 . \\
& + \text { : détecteur } 3 . \\
& + \text { : détecteur } 4 .
\end{aligned}
$$

Courbes théoriques en traits pleins. créneau, on trace $I_{\mathrm{c}}$ en fonction de la fréquence pour les divers détecteurs. La figure 7 donne les résultats obtenus. L'accord entre les courbes théoriques et les résultats expérimentaux est excellent, sauf pour le détecteur 4.

Il apparaît que les écarts proviennent du fait que, pour ce détecteur, la distinction entre cavité de détection et tube d'entrée n'est plus nette; autrement dit (cf. $\S 3.1), N(t)$ n'est plus homogène dans tout le volume de la cavité. On conçoit que pour que $N(t)$ puisse être considéré comme homogène, il faut un tube d'autant plus long et étroit que $V$ est faible.

Conformément à la théorie, le signal obtenu est d'autant plus grand que la constante du tube $A$ est petite et que le volume est petit. Cependant, l'augmentation du signal obtenue en allongeant le tube n'est appréciable qu'aux très basses fréquences et alors l'intensité du signal ne dépend quasiment pas du volume de détection ( $I_{\mathrm{c}}$ proportionnel à $\left.1 / A\right)$.

Par contre, le tube d'entrée est sans effet aux fréquences plus élevées, le signal devenant proportionnel à $1 / V \omega$ et ne dépendant plus (pour une fréquence donnée) que de $V$.

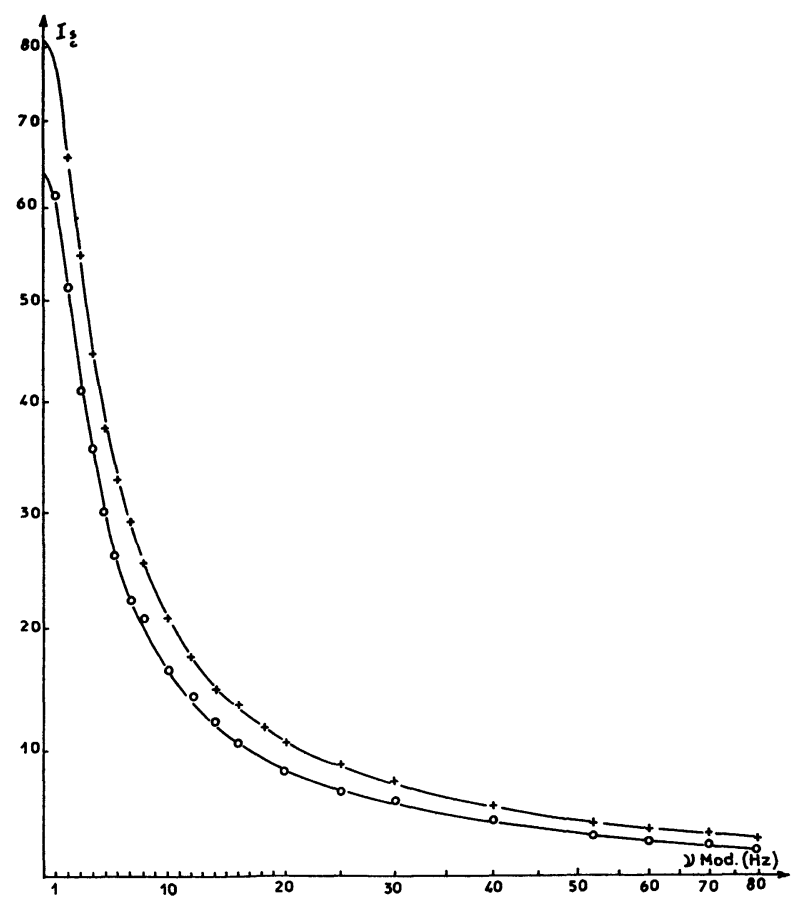

Fig. 8. - Comparaison des modulations sinusoïdale et créneau (détecteur 2):

$$
\begin{aligned}
& + \text { : Modulation créneau. } \\
& \text { : : Modulation sinusoïdale. } \\
& \text { Courbes théoriques en traits pleins. }
\end{aligned}
$$

Le champ HF étant modulé sinusoïdalement, nous vérifions pour le détecteur 2, que $I_{\mathrm{s}}$ et $I_{\mathrm{c}}$ sont dans le rapport $4 / \pi$. L'amplitude du champ $H F$ est alors exceptionnellement fixée à $2,5 \mathrm{mV} / \mathrm{cm}$ de façon à obtenir une modulation sinusoïdale correcte du jet (voir Fig. 4). Les résultats sont représentés sur la figure 8 . 
4.2 RAPPORT SIGNAL SUR BRUIT. - Du point de vue de la sensibilité de la détection, c'est évidemment le rapport signal sur bruit qui fournit le résultat le plus intéressant. Dans le spectromètre que nous utilisons, le bruit provient essentiellement des fluctuations d'intensité du jet moléculaire. Ces fluctuations sont assez lentes et il en résulte un bruit TBF de fréquence centrale certainement inférieure à $1 \mathrm{~Hz}$.

On peut donc s'attendre à ce que le niveau de bruit soit indépendant du volume de la cavité de détection, mais soit par contre proportionnel à la longueur du tube d'entrée. La figure 9 où nous avons porté l'intensité moyenne de bruit $I_{\mathrm{B}}$ en fonction de la fréquence pour les 4 détecteurs vérifie cette hypothèse. Les points se groupent sur 2 courbes, chaque courbe correspondant à un tube d'entrée donné.

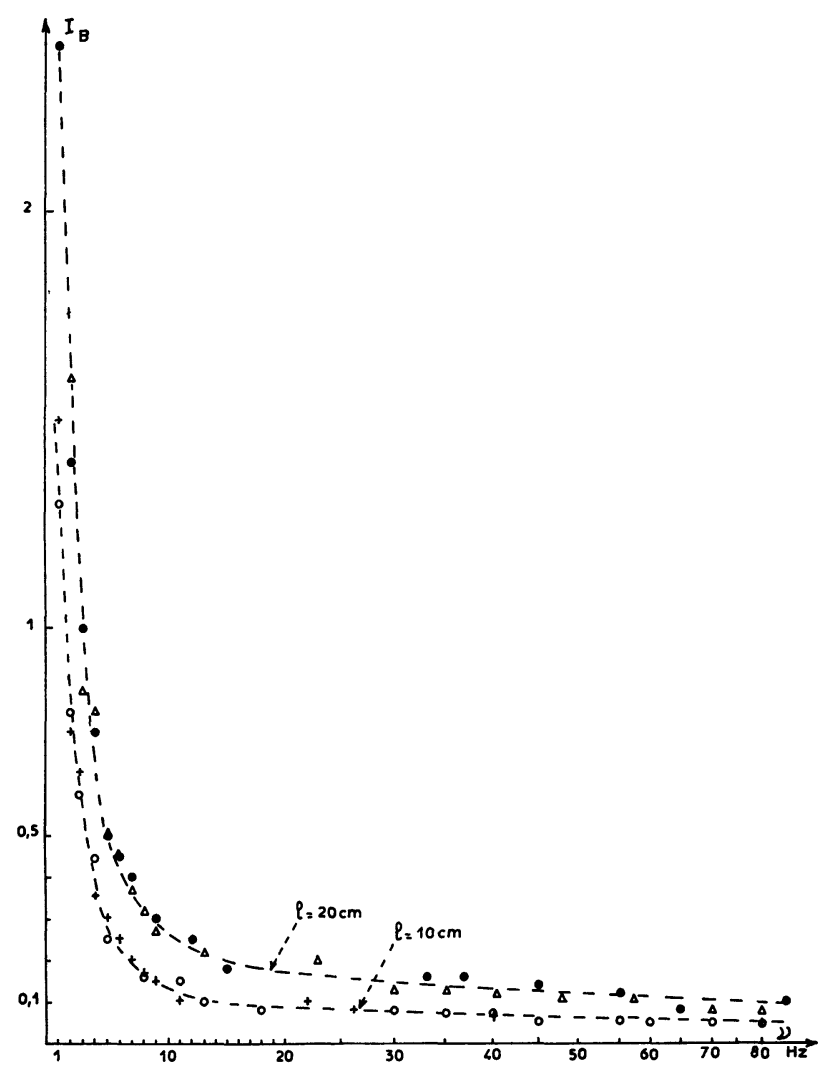

Fig. 9. - Comparaison du bruit pour les 4 détecteurs. Pour les points expérimentaux, même symbolisme que la figure 7 .

La figure 10 nous donne alors les rapports signal/ bruit $\sigma$ pour chaque détecteur. Comme on pouvait s'y attendre, le détecteur convenant le mieux dans notre cas est celui qui associe le tube d'entrée le plus court (niveau du bruit le plus faible) au volume de détection le plus faible (décroissance de $I$ avec la fréquence la plus faible), cette conclusion restant valable pour le détecteur 4 bien que le signal obtenu expérimentalement soit légèrement inférieur au signal théorique pour les fréquences moyennes. Les courbes de rapport signal sur bruit que nous présentons ici ne sauraient être généralisées à un appareillage quelconque. En effet, si la courbe du signal en fonction de la fréquence traduit bien l'intégration de ce dernier par le détecteur et présente un caractère de généralité (dans les limites

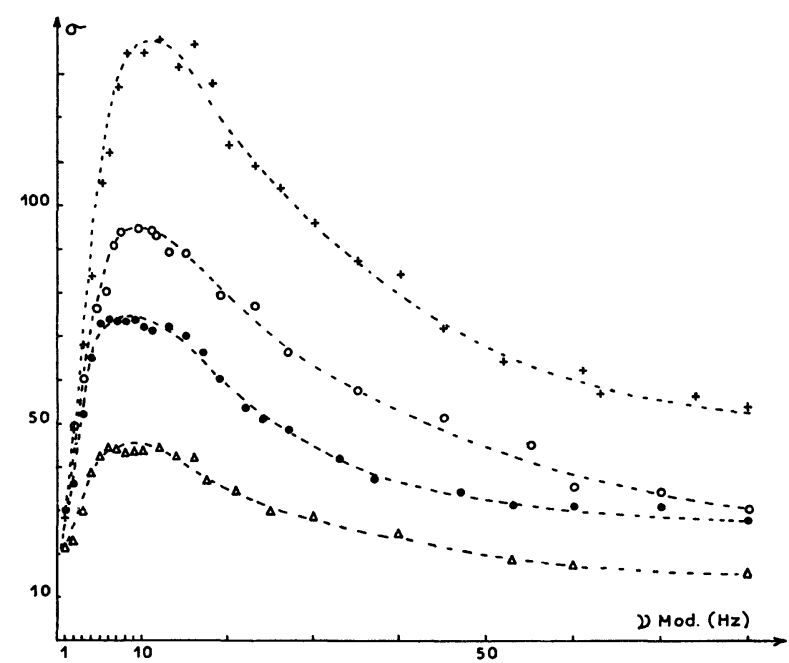

Fig. 10. - Rapport signal sur bruit pour les 4 détecteurs. Même symbolisme que pour les figures 7 et 9 .

précisées ci-dessus), il n'en est pas de même du bruit, l'évolution du niveau de bruit avec la fréquence étant liée à un type de montage particulier.

La connaissance du spectre de bruit et du fonctionnement du détecteur permettrait de déterminer de façon théorique les conditions conduisant au rapport signal sur bruit optimum. En pratique, la connaissance du spectre de bruit est assez malaisée d'une part et, d'autre part, pour le détecteur, on est limité par la validité de la théorie et les possibilités de réalisation pour les valeurs extrêmes des paramètres $V$ et $P$. Dans ces conditions, l'étude de l'optimum du rapport signal sur bruit doit faire une large place à l'expérience.

5. Conclusion. - L'exemple traité 'ci-dessus peut aisément se généraliser à tout type de détecteur ; néanmoins il faut en tirer deux conclusions bien distinctes :

$1^{0}$ Dans un certain domaine (dont les limites peuvent être déterminées expérimentalement) on peut faire une théorie satisfaisante du fonctionnement du déteteur. On peut alors, en tenant compte de l'effet d'intégration, obtenir une théorie plus complète du signal issu d'un dispositif à jet moléculaire. Il est particulièrement important de tenir compte de cet effet d'intégration lorsqu'on s'intéresse à la forme d'un signal, par exemple dans l'étude de la distribution des vitesses par l'intermédiaire de la déformation d'un paquet de molécules de forme initialement connue (le signal final obtenu est alors la convolution de l'extension du paquet de molécules avec la réponse du détecteur).

$2^{\circ}$ La conclusion est moins nette en ce qui concerne l'obtention de la meilleure sensibilité, une étude théorique complète nécessitant conjointement la connaissance du spectre de bruit et un optimum de $\sigma$ situé 
dans le domaine de validité de la théorie du détecteur. le plus performant parmi ceux possédant un faible Cependant, même si ces conditions ne sont pas réalisées, une connaissance sommaire du bruit peut fournir a priori des indications quant à la construction du détecteur. C'est le cas de nos expériences, par exemple, où un bruit $\mathrm{TBF}$ nous conduit à rechercher le détecteur volume et un tube d'entrée court.

Remerciements. - Nous remercions MM. Delafin et Mortreux de l'Ecole Centrale pour leur collaboration aux expériences.

\section{Bibliographie}

[1] Campargue, R., Thèse, Paris, 1970.

[2] Treguier, J. P., Hagene, M., Pasquerault, D., Revue Phys. Appl. 5 (1970) 343.

[3] Berthier, J. P., Constans, A., Daury, G. et Lostis, P., Revue Phys. Appl. 6 (1971) 433.

[4] Hagene, M., Pasquerault, D., Louineau, A., C. R. Hebd. Séan. Acad. Sci. 274B (1972) 1309.
[5] Dyke, T. R., Tomasevitch, G. R., Klemperer, W., FalCONER, W. E., J. Chem. Phys. 57 (1972) 6.

[6] Genty, C., Thèse, Besançon, 1970.

[7] RamseY, N. F., Molecular Beams (Clarendon Press, Oxford), 1956.

[8] Genty, C., Mandret, G., Sungur, N., Etude par détection de phase de la vitesse d'un jet moléculaire, $C . R$. Hebd. Séan. Acad. Sci. 275B (1972) 13. 